\title{
Visualization of Regression Models Using visreg
}

by Patrick Breheny and Woodrow Burchett

\begin{abstract}
Regression models allow one to isolate the relationship between the outcome and an explanatory variable while the other variables are held constant. Here, we introduce an R package, visreg, for the convenient visualization of this relationship via short, simple function calls. In addition to estimates of this relationship, the package also provides pointwise confidence bands and partial residuals to allow assessment of variability as well as outliers and other deviations from modeling assumptions. The package provides several options for visualizing models with interactions, including lattice plots, contour plots, and both static and interactive perspective plots. The implementation of the package is designed to be fully object-oriented and interface seamlessly with R's rich collection of model classes, allowing a consistent interface for visualizing not only linear models, but generalized linear models, proportional hazards models, generalized additive models, robust regression models, and many more.
\end{abstract}

\section{Introduction}

In simple linear regression, it is both straightforward and extremely useful to plot the regression line. The plot tells you everything you need to know about the model and what it predicts. It is common to superimpose this line over a scatter plot of the two variables. A further refinement is the addition of a confidence band. Thus, in one plot, the analyst can immediately assess the empirical relationship between $x$ and $y$ in addition to the relationship estimated by the model and the uncertainty in that estimate, and also assess how well the two agree and whether assumptions may be violated.

Multiple regression models address a more complicated question: what is the relationship between an explanatory variable and the outcome as the other explanatory variables are held constant? This relationship is just as important to visualize as the relationship in simple linear regression, but doing so is not nearly as common in statistical practice.

As models get more complicated, it becomes more difficult to construct these sorts of plots. With multiple variables, we cannot simply plot the observed data, as this does not hold the other variables constant. Interactions among variables, transformations, and non-linear relationships all add extra barriers, making it time-consuming for the analyst to construct these plots. This is unfortunate, however - as models grow more complex, there is an even greater need to represent them with clear illustrations.

In this paper, we aim to eliminate the hurdle of implementation through the development of a simple interface for visualizing regression models arising from a wide class of models: linear models, generalized linear models, robust regression models, additive models, proportional hazards models, and more. We implement this interface in $\mathrm{R}$ and provide it as the package visreg, publicly available from the Comprehensive R Archive Network. The purpose of the package is to automate the work involved in plotting regression functions, so that after fitting one of the above types of models, the analyst can construct attractive and illustrative plots with simple, one-line function calls. In particular, visreg offers several tools for the visualization of models containing interactions, which are among the easiest to misinterpret and the hardest to explain.

It is worth noting that there are two distinct goals involved in plotting regression models: illustrating the fitted model visually and diagnosing violations of model assumptions through examination of residuals. The approach taken by visreg is to construct a single plot that simultaneously addresses both goals. This is not a new idea. Indeed, this project was inspired by the work of Trevor Hastie, Robert Tibshirani, and Simon Wood, who have convincingly demonstrated the utility of these types of plots in the context of generalized additive models (Hastie and Tibshirani, 1990; Wood, 2006).

In particular, visreg offers partial residuals, which can be defined for any regression model and are easily superimposed on visualization plots. Partial residuals are widely useful in detecting many types of problems, although several authors have pointed out that they are not without limitations (Mallows, 1986; Cook, 1993). Various extensions and modifications of partial residuals have been proposed, and there is an extensive literature on regression diagnostics (Belsley et al., 1980; Cook and Weisberg, 1982); indeed, many diagnostics are specific to the type of model (e.g., Pregibon, 1981; Grambsch and Therneau, 1994; Loy and Hofmann, 2013). Partial residuals are a useful, easily generalized idea that can applied to virtually any type of model although it is certainly worth being aware of other types of diagnostics that are specific to the modeling framework in question.

There are a number of $\mathrm{R}$ packages that offer functions for visualizing regression models, including 
rms (Harrell, 2015), rockchalk (Johnson, 2016), car (Fox and Weisberg, 2011), effects (Fox, 2003), and, in base $\mathrm{R}$, the termplot function. The primary advantage of visreg over these alternatives is that each of them is specific to visualizing a certain class of model, usually $1 \mathrm{~m}$ or glm. visreg, by virtue of its object-oriented approach, works with any model that provides a predict method - meaning that it can be used with hundreds of different $R$ packages as well as user-defined model classes. We also feel that visreg offers a simpler interface and produces nicer-looking plots, but admit that beauty is in the eye of the beholder. Nevertheless, there are situations in which each of these packages are very useful and offer some features that others do not, such as greater flexibility for other types of residuals (car) and better support for visualizing three-way interactions (effects).

Each type of model has different mathematical details. All models, however, describe how the response is expected to vary as a function of the explanatory variables. In $\mathrm{R}$, this is implemented for an extensive catalog of models that provide an associated predict method. Although there are no explicit rules forcing programmers to write predict methods for a given class in a consistent manner, there is a widely agreed-upon convention to follow the general syntax of predict. $1 \mathrm{~m}$. It is this abstraction upon which visreg is based: the use of object-oriented programming to provide a single tool with a consistent interface for the convenient visualization of a wide array of models.

There are thousands of $\mathrm{R}$ packages, many of which provide an implementation of some type of model. It is impossible for any programmer or team of programmers to write an $\mathrm{R}$ package that is familiar with the details of all of them. However, the encapsulation and abstraction offered by an object-oriented programming language allow for an elegant solution to this problem. By passing a fitted model object to visreg, we can call the predict method provided by that model class to obtain appropriate predictions and standard errors without needing to know any of the details concerning how those calculations work for that type of model; the same applies to construction of residuals through the residual method.

The only other R package that we are aware of that provides this kind of object-oriented flexibility is plotmo by Stephen Milborrow. The visreg and plotmo projects were each started independently around the year 2011 and have developed into mature, widely used packages for model visualization. The organization and syntax of the packages is quite different, but both are based on the idea of using the generic predict and residuals methods provided by a model class to offer a single interface capable of visualizing virtually any type of model. The primary difference between the two packages is that plotmo separates the visualization of models and the plotting of residuals, constructed using the plotmo() and plotres() functions, respectively, while as mentioned earlier, visreg combines the two into a single plot (plotmo offers an option to superimpose the unadjusted response onto a plot, but this is very different from plotting partial residuals). Furthermore, as one would expect, each package offers a few options that the other does not. For example, plotmo offers the ability to construct partial dependence plots (Hastie et al., 2009), while visreg offers options for contrast plots and what we call "cross-sectional" plots (Figs. 6, 7, and 8). Broadly speaking, plotmo is somewhat more oriented towards machine learning-type models, while visreg is more oriented towards regression models, though both packages can be used for either purpose. In particular, plotmo supports the X, y syntax used by packages like glmnet, which is more popular among machine learning packages, while visreg focuses exclusively on models that use a formula-based interface.

The outline of the paper is as follows. In "Conditional and contrast plots", we explicitly define the relevant mathematical details for what appears in visreg's plots. The remainder of the article is devoted to illustrating the interface and results produced by the software in three extensions of simple linear regression: multiple (additive) linear regression models, models that possess interactions, and finally, other sorts of models, such as generalized linear models, proportional hazards models, random effect models, random forests, etc.

\section{Conditional and contrast plots}

We begin by considering regression models, where all types of visreg plots are well-developed and clearly defined. At the end of this section, we describe how these ideas can be extended generically to any model capable of making predictions.

In a regression model, the relationship between the outcome and the explanatory variables is expressed in terms of a linear predictor $\eta$ :

$$
\eta=\mathbf{X} \beta=\sum_{j} \mathbf{x}_{j} \beta_{j}
$$

where $\mathbf{x}_{j}$ is the $j$ th column of the design matrix $\mathbf{X}$. For the sake of clarity, we focus in this section on linear regression, in which the expected value of the outcome $\mathbb{E}\left(Y_{i}\right)$ equals $\eta_{i}$; extensions to other, nonlinear models are discussed in "Other models". In the absence of interactions (see "Linear models 
with interactions"), the relationship between $X_{j}$ and $Y$ is neatly summarized by $\beta_{j}$, which expresses the amount by which the expected value of $Y$ changes given a one-unit change in $X_{j}$.

Partial residuals are a natural multiple regression analog to plotting the observed $x$ and $y$ in simple linear regression. Partial residuals were developed by Ezekiel (1924), rediscovered by Larsen and McCleary (1972), and have been discussed in numerous papers and textbooks ever since (Wood, 1973; Atkinson, 1982; Kutner et al., 2004). Letting $\mathbf{r}$ denote the vector of residuals for a given model fit, the partial residuals belonging to variable $j$ are defined as

$$
\begin{aligned}
\mathbf{r}_{j} & =\mathbf{y}-\mathbf{X}_{-j} \widehat{\boldsymbol{\beta}}_{-j} \\
& =\mathbf{r}+\mathbf{x}_{j} \widehat{\boldsymbol{\beta}}_{j},
\end{aligned}
$$

where the $-j$ subscript refers to the portion of $\mathbf{X}$ or $\beta$ that remains after the $j$ th column/element is removed.

The reason partial residuals are a natural extension to the multiple regression setting is that the slope of the simple linear regression of $\mathbf{r}_{j}$ on $\mathbf{x}_{j}$ is equal to the value $\widehat{\boldsymbol{\beta}}_{j}$ that we obtain from the multiple regression model (Larsen and McCleary, 1972).

Thus, it would seem straightforward to visualize the relationship between $X_{j}$ and $Y$ by plotting a line with slope $\beta_{j}$ through the partial residuals. Clearly, however, we may add any constant to the line and to $\mathbf{r}_{j}$ and the above result would still hold. Nor is it obvious how the confidence bands should be calculated.

We consider asking two subtly different questions about the relationship between $X_{j}$ and $Y$ :

(1) What is the relationship between $\mathbb{E}(Y)$ and $X_{j}$ given $\mathbf{x}_{-j}=\mathbf{x}_{-j}^{*}$ ?

(2) How do changes in $X_{j}$ relative to a reference value $x_{j}^{*}$ affect $\mathbb{E}(Y)$ ?

The biggest difference between the two questions is that the first requires specification of some $\mathbf{x}_{-j}^{*}$, whereas the second does not. The reward for specifying $\mathbf{x}_{-j}^{*}$ is that specific values for the predicted $\mathbb{E}(Y)$ may be plotted on the scale of the original variable $Y$; the latter type of plot can address only relative changes. Here, we refer to the first type of plot as a conditional plot, and the second type as a contrast plot. As we will see, the two questions produce regression lines with identical slopes, but with different intercepts and confidence bands. It is worth noting that these are not the only possible questions; other possibilities, such as "What is the marginal relationship between $X_{j}$ and $Y$, integrating over $\mathbf{X}_{-j}$ ?" exist, although we do not explore them here.

For a contrast plot, we consider the effect of changing $X_{j}$ away from an arbitrary point $x_{j}^{*}$; the choice of $x_{j}^{*}$ thereby determines the intercept, as the line by definition passes through $\left(x_{j}^{*}, 0\right)$. The equation of this line is $y=\left(x-x_{j}^{*}\right) \widehat{\beta}_{j}$. For a continuous $X_{j}$, we set $x_{j}^{*}$ equal to $\bar{x}_{j}$. The confidence interval at the point $x_{j}=x$ is based on

$$
V(x)=\mathbb{V}\left\{\hat{\eta}(x)-\hat{\eta}\left(x_{j}^{*}\right)\right\}=\left(x-x_{j}^{*}\right)^{2} \mathbb{V}\left(\widehat{\beta}_{j}\right) .
$$

When $X_{j}$ is categorical, we plot differences between each level of the factor and the reference category (see Figure 3 for an example); in this case, we are literally plotting contrasts in the classical ANOVA sense of the term (hence the name). Our usage of the term "contrast" for continuous variables is somewhat looser, but still logical in the sense that it estimates the contrast between a value of $X_{j}$ and the reference value.

For a conditional plot, on the other hand, all explanatory variables are fully specified by $x$ and $\mathbf{x}_{-j}^{*}$. Let $\lambda(x)^{T}$ denote the row of the design matrix that would be constructed from $x_{j}=x$ and $\mathbf{x}_{-j}^{*}$. Then the equation of the line is $y=\lambda(x)^{T} \widehat{\boldsymbol{\beta}}$ and the confidence interval at $x$ is based on

$$
V(x)=\mathbb{V}\left\{\lambda(x)^{T} \widehat{\boldsymbol{\beta}}\right\}=\lambda(x)^{T} \mathbb{V}(\widehat{\boldsymbol{\beta}}) \lambda(x) .
$$

In both conditional and contrast plots, the confidence interval at $x$ is then formed around the estimate in the usual manner by adding and subtracting $t_{n-p, 1-\alpha / 2} \sqrt{V(x)}$, where $t_{n-p, 1-\alpha / 2}$ is $1-\alpha / 2$ quantile of the $t$ distribution with $n-p$ degrees of freedom. Examples of contrast plots and conditional plots are given in Figures 2 and 3. Both plots depict the same relationship between wind and ozone level as estimated by the same model (details given in the following section). Note the difference, however, in the vertical scale and confidence bands. In particular, the confidence interval for the contrast plot has zero width at $x_{j}^{*}$; all other things remaining the same, if we do not change $X_{j}$, we can say with certainty that $\mathbb{E}(Y)$ will not change either. There is still uncertainty, however, regarding the 
actual value of $\mathbb{E}(Y)$, which is illustrated in the fact that the confidence interval of the conditional plot has positive width everywhere.

This description of confidence intervals focuses on Wald-type confidence intervals of the form of estimate \pm multiple of the standard error, constructed on the scale of the linear predictor. This is the most common type of interval provided by modeling packages in $\mathrm{R}$, and the only one for which a widely agreed-upon, object-oriented consensus has emerged in terms of what the predict method returns. For this reason, this is usually the only type of interval available for plotting by visreg. However, it should be noted that these intervals are common for their convenience, not due to superiority; it is typically the case that more accurate confidence intervals exist (see, for example, Efron, 1987; Withers and Nadarajah, 2012). In principle, one could plot other types of intervals, but visreg does not calculate intervals itself so much as plot the intervals that the modeling package returns. Thus, unless the modeling package provides methods for calculating other types of intervals, visreg is restricted to plotting Wald intervals.

Contrast plots can only be constructed for regression-based models, as they explicitly require an additive decomposition in terms of a design matrix and coefficients. Conditional plots, however, can be constructed for any model that produces predictions. Denote this prediction $f(\mathbf{x})$, where $\mathbf{x}$ is a vector of predictors for the model. Writing this as a one-dimensional function of predictor $j$ with the remaining predictors fixed at $\mathbf{x}_{-j}^{*}$, let us express this prediction as $f\left(x \mid \mathbf{x}_{-j}^{*}\right)$. In a conditional plot, the partial residuals for predictor $j$ are

$$
\begin{aligned}
\mathbf{r}_{j} & =\mathbf{r}+x_{j} \widehat{\beta}_{j}+\mathbf{x}_{-j}^{*} \widehat{\boldsymbol{\beta}}_{-j} \\
& =\mathbf{r}+f\left(x \mid \mathbf{x}_{-j}^{*}\right),
\end{aligned}
$$

which offers a clear procedure for constructing the equivalent of partial residual for general prediction models. Note that this construction requires the model class to implement a residuals method. If a model class lacks a residuals method, visreg will still produce a plot, but must omit the partial residuals; see "Non-regression models" for additional details. Likewise, visreg requires the predict method for the model class to return standard errors in order to plot confidence intervals; see "Hierarchical and random effect models" for an example in which standard errors are not returned.

It is worth mentioning that visreg is only concerned with confidence bands for the conditional mean $\mathbb{E}(Y \mid \mathbf{X})$, not "prediction intervals" that have a specified probability of containing a future outcome $Y$ observed for a certain value of $\mathbf{X}$. Unlike standard errors for the mean, very few model classes in R offer methods for calculating such intervals - indeed, such intervals are often not welldefined outside of classical linear models.

\section{Additive linear models}

We are now ready to describe the basic framework and usage of visreg. In this section, we will fit various models to a data set involving the relationship between air quality (in terms of ozone concentration) and various aspects of weather in the standard $\mathrm{R}$ data set airquality.

\section{Basic framework}

The basic interface to the package is the function visreg, which requires only one argument: the fitted model object. So, for example, the following code produces Figure 1:

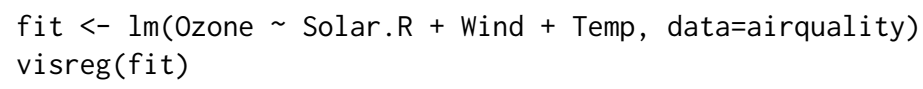

By default, visreg provides conditional plots for each of the explanatory variables in the model. For the conditioning, the other variables in $\mathbf{x}_{-j}^{*}$ are set to their median for numeric variables and to the most common category for factors. All of these options can be modified by passing additional arguments to visreg. For example, contrast plots can be obtained with the type argument; the following code produces Figure 2.

$$
\begin{aligned}
& \text { visreg(fit, "Wind", type="contrast") } \\
& \text { visreg(fit, "Wind", type="conditional") }
\end{aligned}
$$

The second argument specifies the explanatory variable to be visualized; note that the right plot in Figure 2 is the same as the middle plot in Figure 1.

In addition to continuous explanatory variables, visreg also allows the easy visualization of differences between the levels of categorical variables (factors). The following block of code creates a 

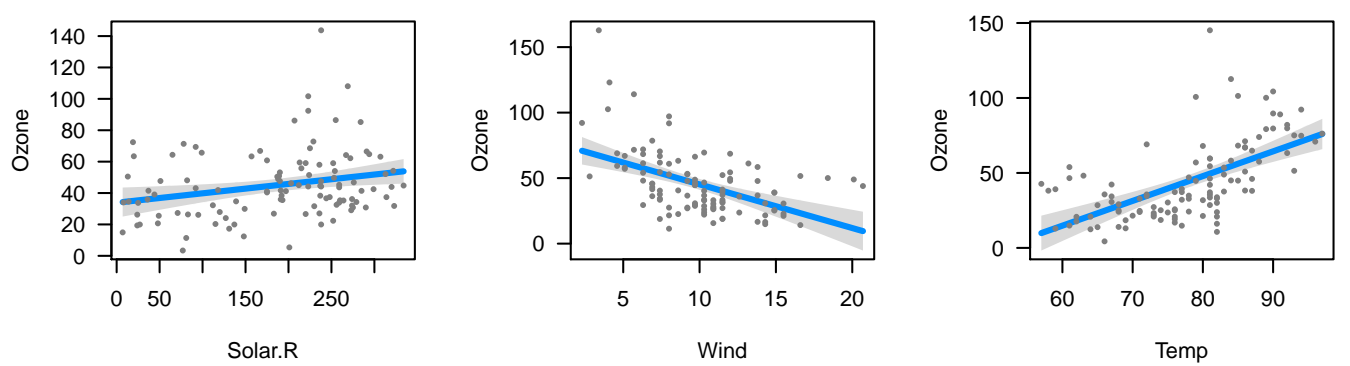

Figure 1: Basic output of visreg for an additive linear model: conditional plots for each explanatory variable.
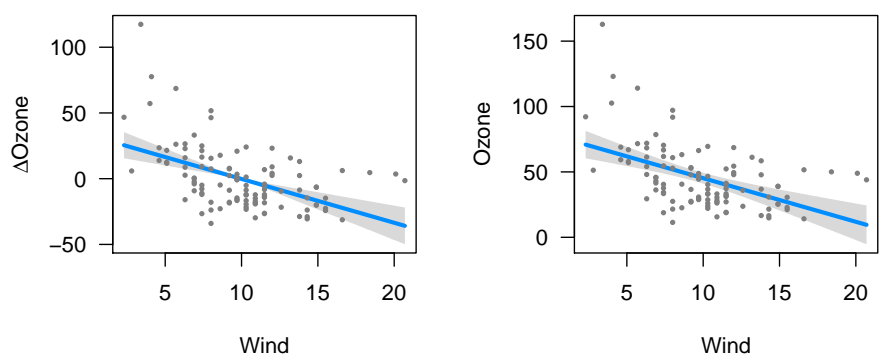

Figure 2: The estimated relationship between wind and ozone concentration in the same model, as illustrated by two different types of plots. Left: Contrast plot. Right: Conditional plot.

factor called Heat by discretizing Temp, and then visualizes its relationship with Ozone, producing the plot in Figure 3.
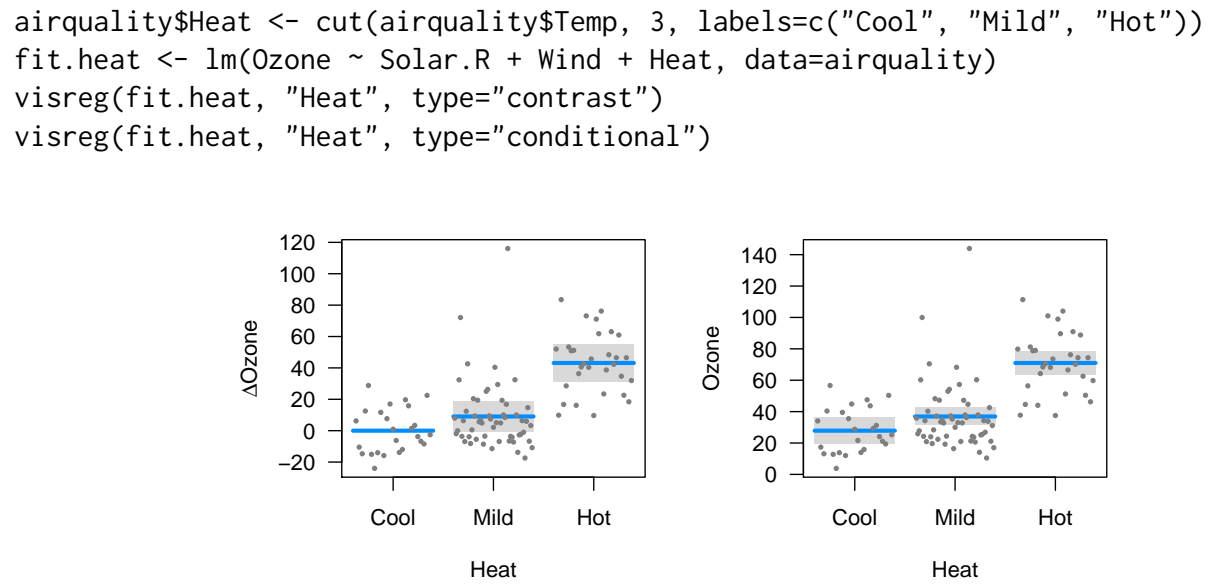

Figure 3: Visualization of a regression function involving a categorical explanatory variable. Left: Contrast plot. Right: Conditional plot.

Again, note that the confidence interval for the contrast plot has zero width for the reference category. There is no uncertainty about how the expected value of ozone will change if we remain at the same level of Heat; it is zero by definition. On the other hand, the width of the confidence interval for Mild heat is wider for the contrast plot than it is for the conditional plot. There is less uncertainty about the expected value of ozone on a mild day than there is about the difference in expected values between mild and cool days. 


\section{Transformations}

Often in modeling, we introduce transformations of explanatory variables, transformations of the response variable, or both. The visreg package automatically handles these transformations when visualizing the regression model.

Linear models assume a linear relationship between the explanatory variables and the outcome. A common way of extending the linear model is to introduce transformations of the original explanatory variables. For example, to allow the effect of wind on ozone to be nonlinear, we may introduce a quadratic term for wind into the model:

fit1 <- Im(Ozone Solar.R + Wind + I(Wind^2) + Temp, data=airquality)

Transformations of the response are also common. For example, ozone levels must be positive. However, as Figure 1 illustrates, a standard linear model allows the estimated relationship and its confidence band to fall below 0 . One way of remedying this is to model the log of ozone concentrations instead of the ozone concentrations directly:

fit2 <- $\operatorname{lm}(\log ($ Ozone $) \sim$ Solar.R + Wind + Temp, data=airquality)

And of course, these elements may be combined:

fit3 $<-\operatorname{lm}\left(\log (\right.$ Ozone $) \sim$ Solar.R + Wind $+I\left(\right.$ Wind^$\left.^{\wedge} 2\right)+$ Temp, data=airquality $)$

Visualization is particularly important in these models, as it is difficult to determine the exact nature of the relationship between explanatory variable and response simply by looking at the regression coefficients when that relationship is nonlinear. The visreg package provides a convenient way to view such relationships. Transformations involving explanatory variables are handled automatically, while transformations involving the response require the user to provide the inverse transformation. The following code produces Figure 4.

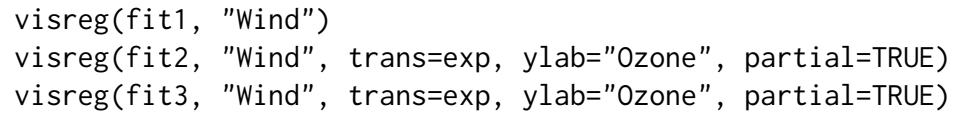

By default, visreg suppresses partial residuals when trans is specified, as this can provide a distorted view of outliers (a mild outlier can become an extreme outlier once a transformation has been applied, and vice versa), but we include them here by explicitly specifying partial=TRUE.
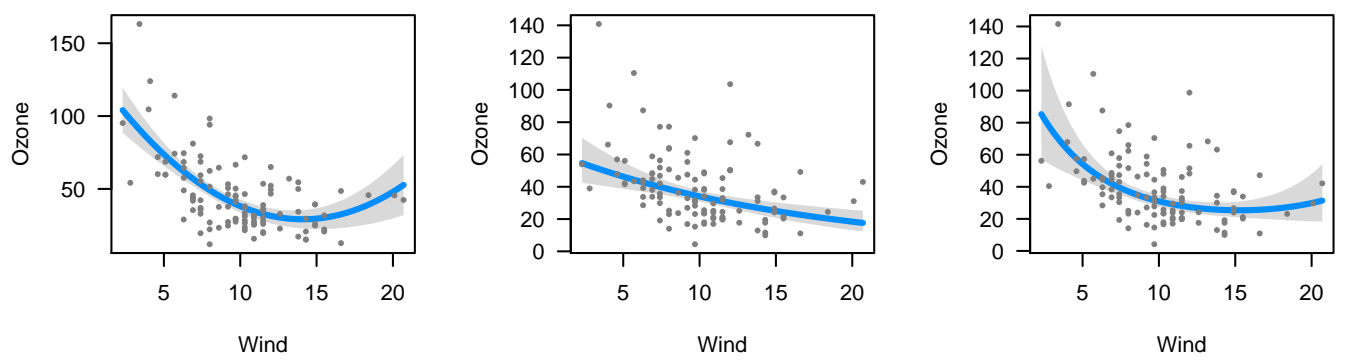

Figure 4: Plots of the modeled relationship between wind and ozone concentration, as estimated by different models. Left: The model contains a transformation of wind. Middle: The model contains a transformation of ozone concentration. Right: The model contains transformations of both wind and ozone.

\section{Conditioning}

As noted in "Basic framework", the default behavior of visreg when constructing a conditional plot is to fill in $\mathbf{x}_{-j}^{*}$ with the median for continuous variables and the most common category for categorical variables. This behavior can be modified using the cond argument. Note that this has no bearing on contrast plots in additive models, which do not require a full specification of $\mathbf{x}_{-j}^{*}$.

The cond argument must be provided as a named list. Each element of that list specifies the value for an element of $\mathbf{x}_{-j}^{*}$; any elements left unspecified are filled in with the median/most common category. We revisit our initial model from "Basic framework" with this code, which produces Figure 5. 
visreg (fit, "Wind", cond=list (Temp=50))

visreg(fit, "Wind")

visreg(fit, "Wind", cond=list $($ Temp=100))
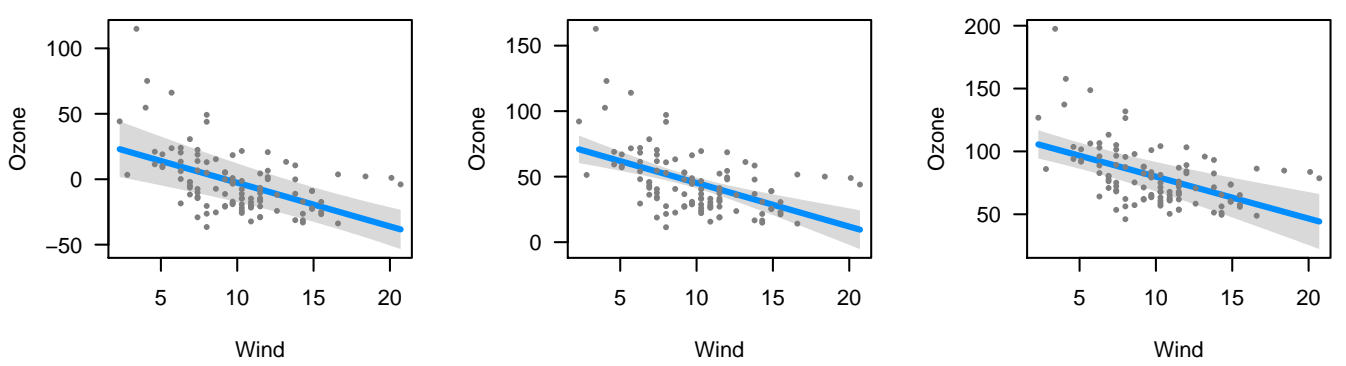

Figure 5: Estimated relationship between wind and ozone concentration, conditioning on different values of temperature. Left: Temperature $=50^{\circ} \mathrm{F}$. Middle: The median temperature, $79^{\circ} \mathrm{F}$ (default). Right: Temperature $=100^{\circ} \mathrm{F}$.

We make several observations concerning Figure 5: i) The values on the vertical axis differ; as we condition on higher temperatures, the expected ozone concentration goes up since the regression coefficient for temperature is positive. ii) The slope of the line, the distance from the line to each residual, and the range of the residuals is the same in all three plots; conditioning on different values of temperature merely adds a constant to the regression line and the partial residuals. iii) The width of the confidence band does change, however: the data set has few observations at very high and very low temperatures, so the standard errors are much larger for the plots on the right and left than for the plot in the middle. iv) The shape of the confidence band also changes. In the middle plot, the confidence band is narrowest in the middle and wider at the ends. In the left plot (conditioning on low temperature), however, the confidence band is narrowest for high wind levels. This arises because there is a negative correlation between wind and temperature $(\hat{\rho}=-0.46)$, and thus, more cold windy days in the data set than cold calm days. The opposite phenomenon happens in the right plot, where the relative absence of hot windy days causes the confidence band to be wider for high winds than for low winds.

Recall that this model had three explanatory variables; in the above example, visreg calculated the conditional response by filling in solar radiation with its median value, as it was not specified otherwise in the cond argument.

\section{Linear models with interactions}

Visualization is also very important for models with interactions - as with polynomial terms, in these models the relationship between an explanatory variable and the response depends on multiple regression coefficients, and a model's fit is more readily understood with a visual representation than by looking at a table of regression coefficients.

For models with interactions, we must simultaneously visualize the effect of two explanatory variables. The visreg package offers two methods for doing this: cross-sectional plots, which plot one-dimensional relationships between the response and one predictor for several values of another predictor, and surface plots, which attempt to provide a picture of the regression surface over both dimensions simultaneously.

\section{Cross-sectional plots}

To begin, let's fit a model that involves an interaction between a continuous term and a categorical term, using our derived variable Heat from "Basic framework":

fit $<-\operatorname{lm}($ Ozone $\sim$ Solar.R + Wind * Heat, data=airquality)

The visreg package creates cross-sectional plots using, by default, the lattice package (Sarkar, 2008). To request a cross-sectional plot, the user specifies a by variable, as in the following code which produces Figure 6.

visreg(fit, "Wind", by="Heat") 


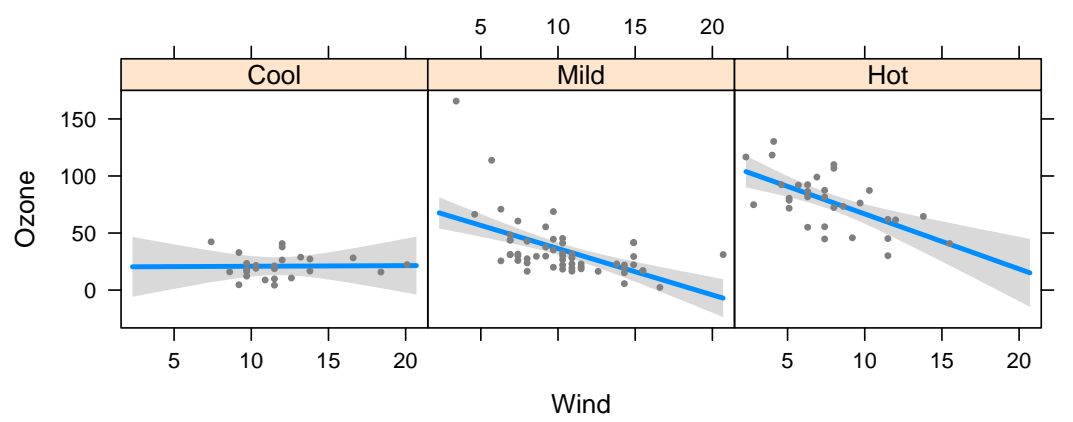

Figure 6: Cross-sectional plots depicting the fit of a model with an interaction between a continuous term (Wind) and a categorical term (Heat), with the continuous term on the horizontal axis.

Alternatively, one can use ggplot2 (Wickham, 2009) as the plotting engine using the option gg=TRUE, as in the following code which produces Figure 7.

visreg(fit, "Wind", by="Heat", gg=TRUE)

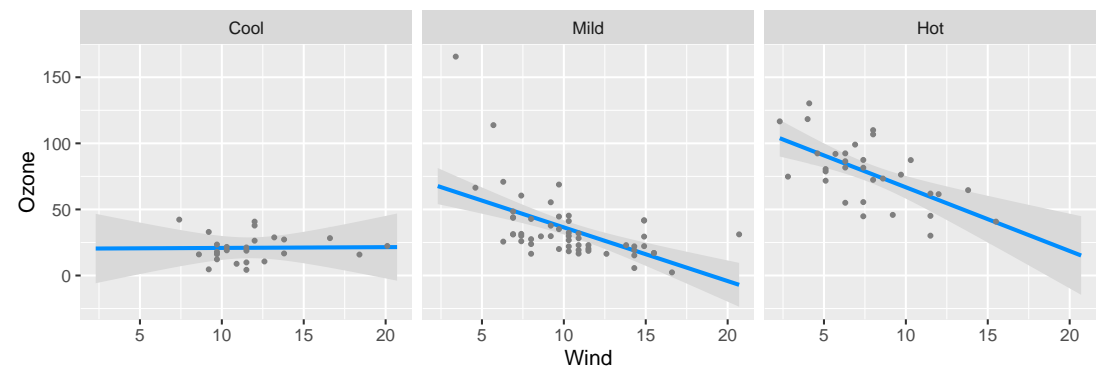

Figure 7: Same as Figure 6, but using ggplot2 as the plotting engine.

The cross-sectional plots in either Figure 6 or 7 allow us to see that the relationship between wind and ozone concentration appears to become more pronounced depending on how hot the day is. On cool days, wind has no effect on ozone concentration. Wind has a moderate effect on ozone concentrations on mild days, and an even larger effect on hot days.

Note that visreg handles the partial residuals properly - the partial residuals for observations collected on cool days appear only in the left panel, and so on. As with the earlier plots, this ensures that the least squares line drawn through the residuals on the plot will yield the same slope as that estimated by the full model fit. Furthermore, this allows us to see potentially influential observations like the one in the middle panel, which has very low wind and very high ozone concentration. Finally, the proper handling of partial residuals also allows us to observe the lack of hot windy days and cool days with no wind that we commented on in "Conditioning". Note that the confidence intervals in these regions are comparatively wide.

Alternatively, we may wish to overlay these cross-sections. This allows for a more direct comparison between the different regression lines, although it often becomes difficult to include partial residuals and confidence bands without crowding the figure. The visreg package allows an overlay option for creating these plots:

visreg(fit, "Wind", by="Heat", overlay=TRUE, partial=FALSE)

The above code produces Figure 8, where the plotting of partial residuals has been turned off for the sake of clarity (similarly, band=FALSE can be specified to turn off the confidence bands). If partial=TRUE, the partial residuals are colored according to the existing scheme.

The above examples featured a continuous variable along the horizontal axis and a categorical variable as the by variable. However, visreg allows each of these variables to be either continuous or categorical. For example, let us try plotting the same model, but reversing the roles of Heat and Wind (Figure 9). 


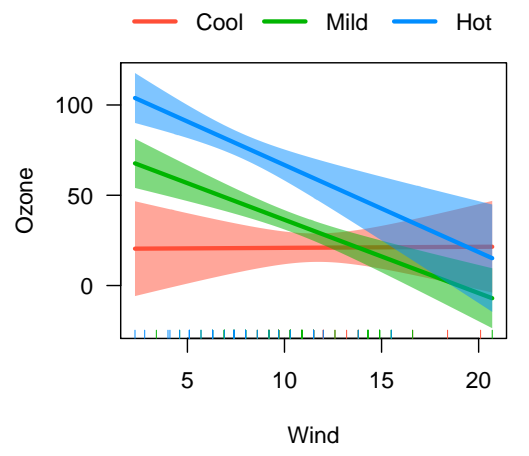

Figure 8: Cross-sectional plot depicting the fit of a model with an interaction between a continuous term (Wind) and a categorical term (Heat), where the regression lines for each category are overlaid.

visreg(fit, "Heat", by="Wind")

The model is the same, but the emphasis of the plot is now on heat instead of wind. Figure 9 illustrates that heat has a pronounced effect on ozone concentration when the day is not windy, but a relatively insignificant effect on ozone for windy days.

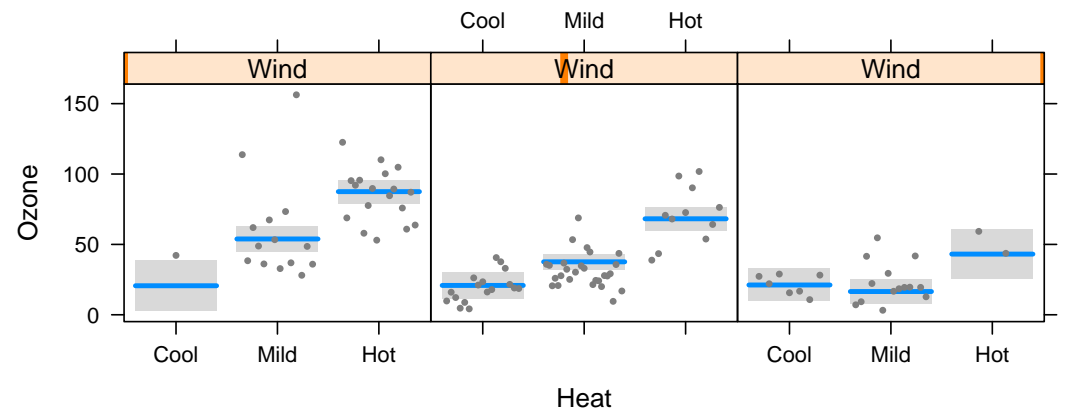

Figure 9: Cross-sectional plots depicting the fit of a model with an interaction between a continuous term (Wind) and a categorical term (Heat), with the categorical term on the horizontal axis.

In contrast to Figure 6, where it was natural to construct a panel for each level of the categorical variable, Figure 8 requires arbitrary decisions concerning how many cross-sections to take, and where to place them. The default behavior of visreg is to take cross-sections at the 10th, 50th, and 90th percentiles of the by variable, although both the number of points and their location can be modified using the breaks option. Again, each residual appears only once, in the panel it is closest to. However, the least squares estimates are no longer equivalent to drawing a line through the partial residuals due to the continuous manner in which information is pooled across the panels.

We have been focusing here on conditional plots, but contrast plots can be made as well by specifying type="contrast". It is worth noting that for a model containing an interaction, a basic call to visreg (i.e., without a by argument) amounts to plotting a main effect in the presence of an interaction. Because this has the potential to be misleading, visreg by default prints a message warning the user of this and reminding him or her of the levels of the other variables at which the plot is constructed. For example, since "Mild" is the most common level of Heat, visreg (fit, "Wind") will produce the middle panel of Figure 6 . The left and right panels, respectively, could be produced by passing Heat="Cool" and Heat="Hot" to the cond argument.

\section{Surface plots}

Another approach to visualizing models with interactions is plotting the regression surface using contour or perspective plots. Suppose we fit a complicated model involving a multiplicative interaction between two-degree-of-freedom natural spline terms for wind and temperature (the function ns is from the splines () package): 
fit <- $\operatorname{lm}($ Ozone Solar.R +ns(Wind, $d f=2) * n s(T e m p, d f=2)$, data=airquality)

Putting aside the question of whether or not this is a good model for analyzing these data, our purpose here is to show that it is difficult to grasp the fit of the model by looking at the regression coefficients directly, but easy to do so using visreg. In addition to the tools for creating cross-sectional plots described in the "Cross-sectional plots", the visreg package provides the function visreg2d, which can be used to produce two-dimensional contour and perspective plots. The following code produces Figure 10:
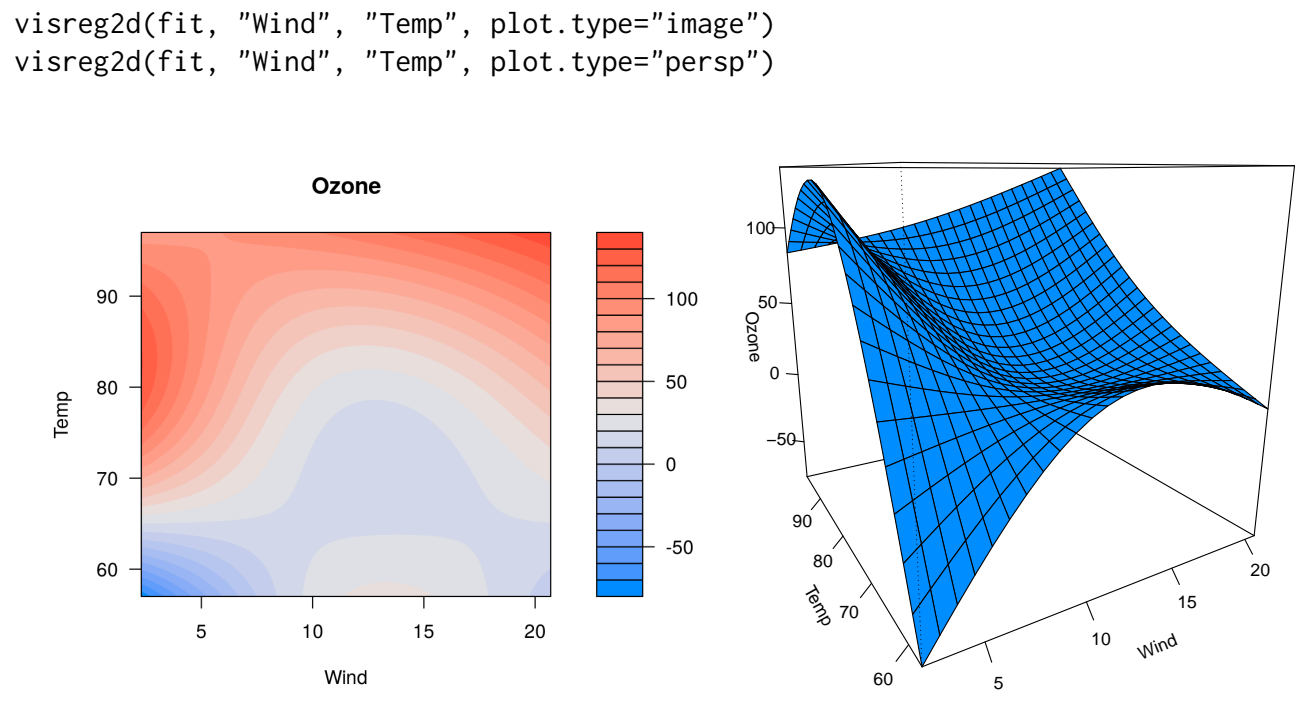

Figure 10: Representations of the regression surface as a function of wind and temperature. Left: Filled contour plot. Right: Perspective plot.

The advantage of these kinds of plots compared with those in "Cross-sectional plots" are that they allow us to visualize the effect of simultaneously varying two factors. The disadvantage is that there is no convenient way of superimposing either residuals or confidence intervals. These plots are most useful when both variables are continuous, as one is not forced to take cross-sections over a continuous variable. The visreg2d function still functions correctly when one or both of its arguments is a categorical variable, although in our opinion, the cross-section plots of "Cross-sectional plots" are more useful in these settings.

In addition to the static perspective plot presented above, visreg $2 \mathrm{~d}$ can also create interactive perspective plots using the rgl package (Adler and Murdoch, 2011), which allow the user to rotate, tilt, and spin the regression surface. This makes it considerably easier to comprehend its three-dimensional shape. Such plots can be constructed with the code:

visreg2d(fit, $x=$ "Wind", $y=$ "Temp", plot.type="rgl")

Visualization of higher-order interactions, such as three-way or four-way interactions, becomes increasingly difficult. To some extent, visreg facilitates visualization of such models through the use of the cond argument. For example, code such as the following could be used to visualize a three-way interaction:

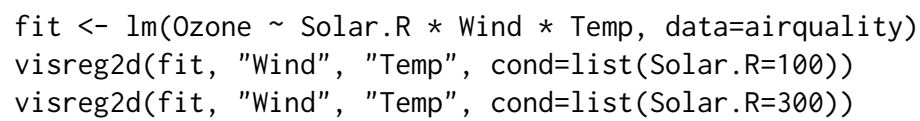

\section{Other models}

As mentioned at the outset, the goal in creating the visreg package was to implement visualization in an object-oriented manner, so that it works with as many classes of models from different functions and packages as possible. All that it requires is functioning model. frame and predict methods for the fitted model object (plotting of partial residuals requires a residuals method as well). Thus, the visreg package and all its options work not only with linear model objects produced by $1 \mathrm{~m}$, but with generalized linear models produced by glm, proportional hazards models produced by coxph (Therneau, 2012), robust linear models produced by rlm (from MASS: Venables and Ripley, 2002), 
negative binomial models produced by glm. nb (from MASS), generalized additive models produced by gam (from mgcv: Wood, 2012), local regression models produced by loess and locfit (Loader, 2010), and many more. Indeed, the type of object does not even need to be part of an R package; user-defined model classes can also be visualized with visreg, provided that they are compatible with model. frame and predict. In this section, we briefly illustrate the use of visreg with some of the above types of models.

\section{Generalized linear models}

We begin with a logistic regression model applied to a study investigating risk factors associated with low birth weight (Hosmer and Lemeshow, 2000). The following code produces Figure 11.
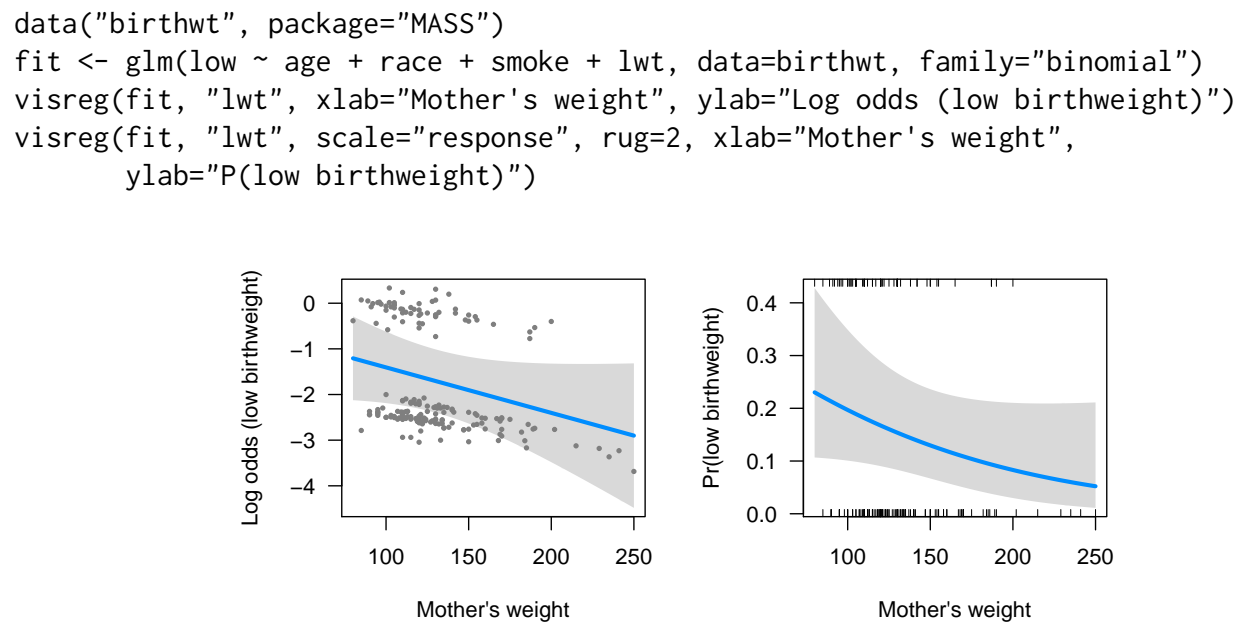

Figure 11: Visualization of a logistic regression model. Left: Log odds scale. Right: Probability scale.

On the left side of Figure 11, the model is plotted on the scale of the linear predictor (the default scale in visreg), where the model is indeed linear. The confidence intervals in the figure are Wald confidence intervals based on standard errors returned by predict.glm. The partial residuals are calculated based on Equation 2, with $\mathbf{r}$ the deviance residuals (the default residuals returned by residuals.glm). The plot on the right is simply a transformed version of the plot on the left, where an inverse logistic transformation has been applied to the regression line and confidence bands (this is handled automatically by the scale="response" option).

Note that for the plot on the right, we have opted to plot a rug as opposed to the partial residuals. The visreg package provides two types of rug annotations. With rug=TRUE or rug=1, a standard rug along the bottom of the plot is provided. With rug=2, separate rugs are drawn on the top for observations with positive residuals and on the bottom for observations with negative residuals (for logistic regression, this corresponds to $Y=1$ and $Y=0$, respectively).

In practice, we have found plots like those on the left useful for visualizing the model fit and observing potential departures from model assumptions such as outliers and influential points, and plots like those on the right very useful for communicating modeling results to non-statisticians.

\section{Other regression models}

Here, we provide a brief demonstration applying visreg to some other types of models (note that these are models for which the effects package is incompatible): a proportional hazards model, a robust regression model, and a local regression model. The left side of Figure 12 presents a visualization of the following proportional hazards model:

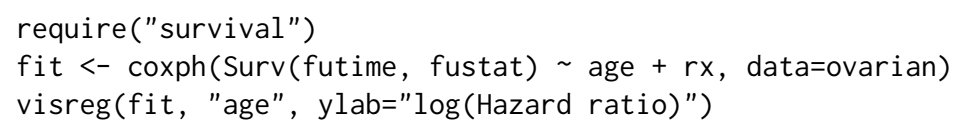

Note that in proportional hazards models, baseline hazard functions are not explicitly estimated, and therefore the meaning behind a conditional plot is questionable. For this reason, contrast plots are (arguably) more appropriate. A similar phenomenon occurs with logistic regression applied to 

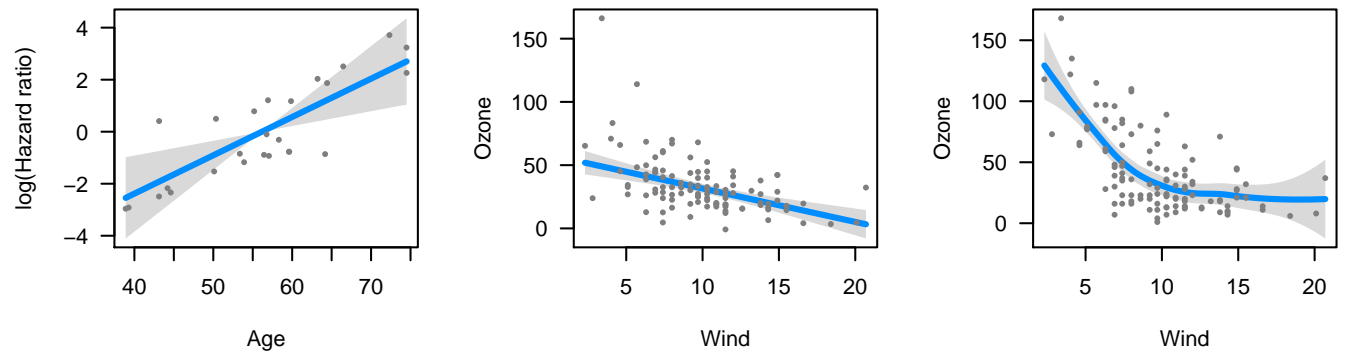

Figure 12: Visualizations of proportional hazards (left), robust regression (middle), and loess (right) models.

case-control studies, in which an intercept is estimated, but is the estimate is biased by the study design.

The middle of Figure 12 presents a visualization of the following robust regression model (using rlm from the MASS package):

fit <- rlm(Ozone Solar.R + Wind * Heat, data=airquality)

visreg(fit, "Wind", ylab="Ozone")

Note that the design matrix for the robust regression model is the same as that from "Crosssectional plots", and that the plot in the middle of Figure 12 is analogous to the middle panel from Figure 6. Note, however, that the robust regression model produces a different fit, due in part to the reduced impact of the potential outlier mentioned in "Cross-sectional plots". Specifically, the fit produced by the robust regression model is flatter and does not predict negative ozone concentrations for high wind levels as the linear regression model does.

Finally, we apply visreg to a local regression model fit with loess, producing a much more useful visualization of the model than the default plot method for loess. This plot appears on the right side of Figure 12.

fit $<-$ loess(Ozone Wind, airquality)

visreg(fit, "Wind", ylab="Ozone")

All of the features and options we mentioned earlier; in particular cross-section and surface plots work in the same way for nonlinear models as they do for linear models.

Computationally, the extension of visreg to nonlinear models is straightforward due to its objectoriented implementation, but it is worth making some comments about partial residuals for nonlinear models. In particular, it is no longer the case that the regression line through the partial residuals produces a line with the same slope as that produced by the model. Viewing nonlinear models as reweighted least squares models, the observations have different weights and these weights are not reflected in the partial residuals plotted by visreg. This phenomenon has been commented on by many authors, with a variety of proposals for alternative types of reweighted partial residuals that may be better at detecting outliers and influential observations (Pregibon, 1981; Landwehr et al., 1984; O'Hara Hines and Carter, 1993).

\section{Non-regression models}

Moving even further from linear models, visreg is also compatible with modeling frameworks that are not even regression-based, such as random forests and support vector machines. Such methods are often thought of as "black boxes", but visreg offers a convenient way to visualize the resulting fit and possibly gain some insight into the model. The following code fits each of the aforementioned models to the airquality data using the randomForest (Breiman et al., 2015) and e1071 (Meyer et al., 2017) packages, and plots the resulting estimated association between ozone and temperature (Figure 13). Some of these packages do not automatically handle missing data, so we first create a complete-case data set aq:

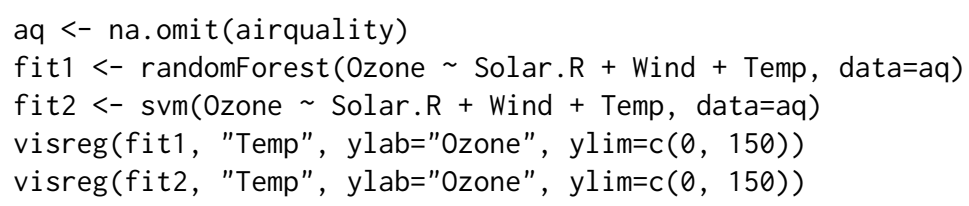



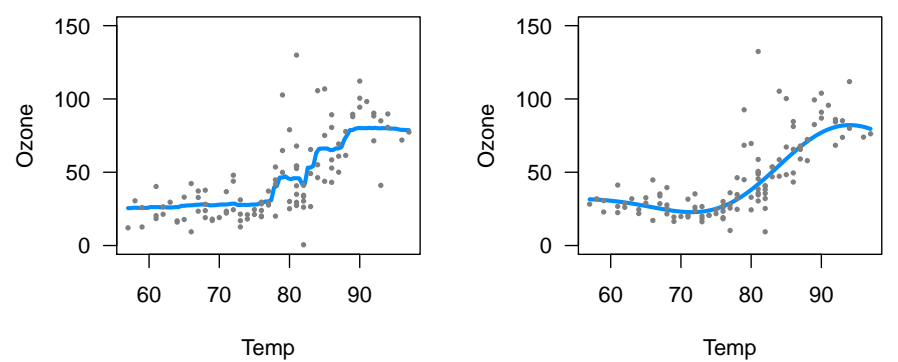

Figure 13: Left: Random forest. Right: Support vector machine.

Both of the results in Figure 13 appear reasonable with the default settings employed, although neither of these models is able to provide confidence bands for fitted values, so no shaded bands appear. A useful feature of plotting the model's predictions, however, is that it illustrates the effect of changing those settings. For example, consider the application of gradient boosting machines to this same data using the gbm package (Ridgeway, 2017). First, it is worth noting that the gbm package does not offer a residuals method. This would normally cause visreg to omit plotting the partial residuals. However, we can supply our own user-defined residuals method, which enables visreg to produce the plots in Figure 14.
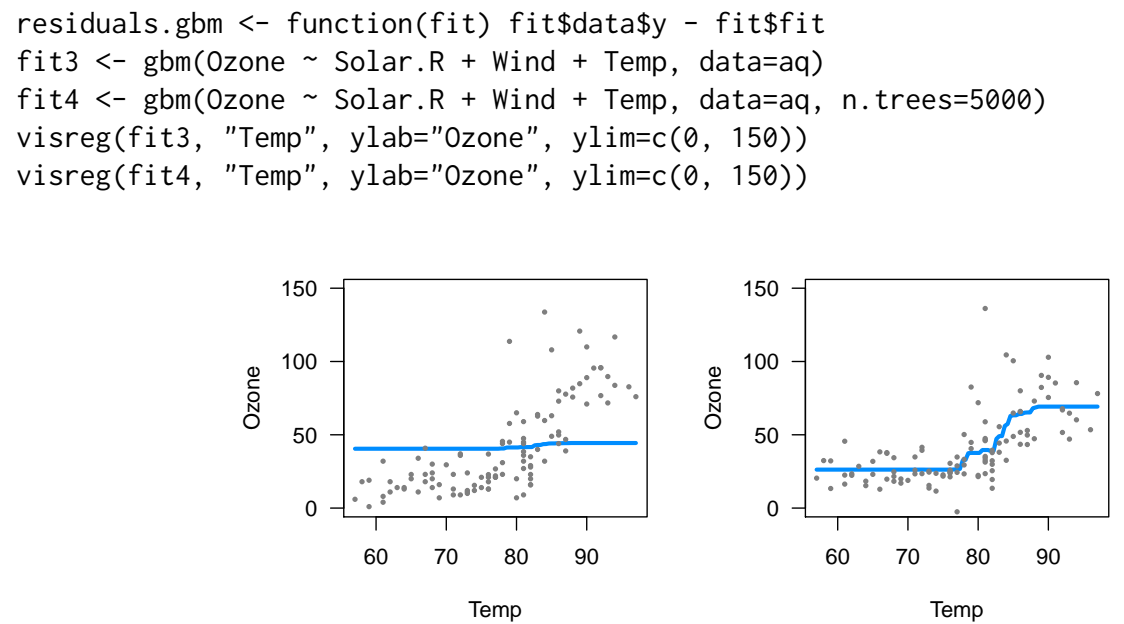

Figure 14: Visualizations of gradient boosting machine. Left: Default setting (100 trees) Right: 5,000 trees.

Note that the default settings for gbm do not produce a very good fit here. In particular, the default number of trees (100) is too low to capture the relationship between temperature and ozone. By increasing the number of trees (to 5,000), we obtain a much more reasonable fit.

\section{Hierarchical and random effect models}

The ability of visreg to visualizing mixed effect models is hindered by the fact that incorporating uncertainty about random effects into predictions is difficult from a frequentist perspective and most $\mathrm{R}$ packages for such models do not offer confidence intervals for such estimates. Nevertheless, visreg is still useful for visualizing the effects of fixed effects in such models using contrast plots, as well as plotting effects without confidence intervals.

As an illustration, we consider a study involving the protein content of cows' milk in the weeks following calving (Diggle et al., 2002). Consider the following random-intercept, random-slope model, fit using the lme4 package (Bates et al., 2012), which also contains a fixed effect for the type of diet each cow was fed.

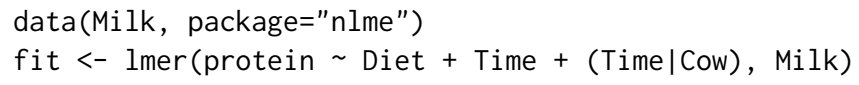


In the lme4 package, the predict method does not return standard errors. This means that any conditional plots constructed by visreg will lack confidence intervals, like those in Figures 13 and 14. This is another example of a situation where a contrast plot is useful: by considering the effect of changing diet while other terms remain constant, the random effects drop out of the model and standard errors/confidence intervals are straightforward, as illustrated in Figure 15. The following code also illustrates how to change graphical options, as there is considerable overplotting of the partial residuals under the default settings.

visreg(fit, "Diet", type="contrast", ylab=expression(Delta*'Protein'), points.par=list (col="\#55555540", cex=0.25))

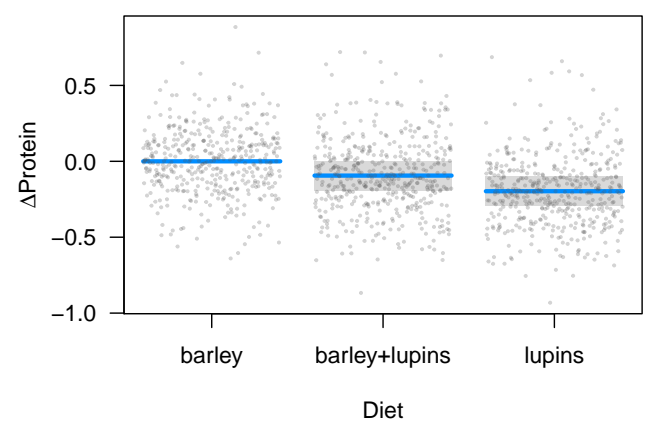

Figure 15: Contrast plot illustrating the fixed effect of diet in the Milk example.

The visreg package can also be used to plot random effects, although as mentioned earlier, the plots will not include intervals. Below, we provide code to plot the modeled relationship between protein content and time. Two aspects of the code are worth pointing out. First, note that according to the object-oriented design of visreg, the predict method supplied by lme4 will used. It has its own option, re. form, to control how random effects are used in the prediction, and this must be passed through visreg accordingly. Second, for the sake of space we subset the plot to ten cows rather than all 79. This can be accomplished by returning, then subsetting, the raw visreg object prior to plotting. Returning the data frames, estimates, confidence intervals, and residuals used in the construction of its plots like this allows users to write their own extensions and modifications of visreg plots.
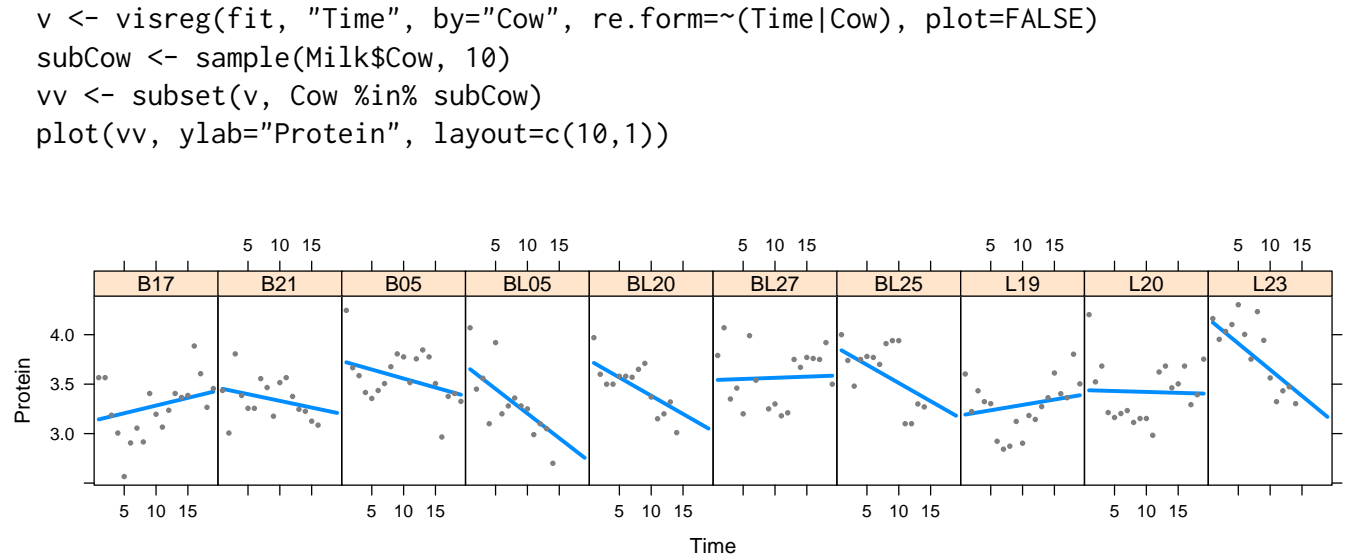

Figure 16: Subject-specific conditional plots for ten randomly chosen cows from the Milk example illustrating the change in protein content over time.

\section{Conclusion}

Partial residuals and how useful they are in detecting influential observations and departures from model assumptions depends on the model. Other types of plots, such as added variable plots 
(Atkinson, 1985), are also helpful for visualizing regression models and their fit. We feel that the approach provided by visreg is reasonable and the best that can be expected from an object-oriented tool that can be applied generically to a wide variety of models, although we certainly acknowledge that other types of plots and visualizations may offer useful additional information for certain types of models.

The visreg package provides a very useful set of tools for simultaneously visualizing the estimated relationship between an explanatory variables and the outcome, the variability of that estimate, and the observations from which the estimates derive. These tools have a simple interface and are readily applied in an object-oriented manner to wide variety of models. We have found the development of this package to provide a convenient and versatile tool to assist with regression modeling, both for model exploration and for communicating modeling results.

More information about visreg, illustrating its various options with numerous examples can be found at http://pbreheny.github.io/visreg.

\section{Bibliography}

D. Adler and D. Murdoch. Rgl: 3D Visualization Device System (OpenGL), 2011. R package version 0.92.798. [p65]

A. C. Atkinson. Regression diagnostics, transformations and constructed variables. Journal of the Royal Statistical Society B, 44:pp. 1-36, 1982. [p58]

A. C. Atkinson. Plots, Transformations, and Regression. Oxford University Press, 1985. [p70]

D. Bates, M. Maechler, and B. Bolker. Lme4: Linear Mixed-Effects Models Using S4 Classes, 2012. R package version 0.999999-0. [p68]

D. A. Belsley, E. Kuh, and R. E. Welsch. Regression Diagnostics. John Wiley \& Sons, 1980. [p56]

L. Breiman, A. Cutler, A. Liaw, and M. Wiener. randomForest: Breiman and Cutler's Random Forests for Classification and Regression, 2015. R package version 4.6-12. [p67]

R. D. Cook. Exploring partial residual plots. Technometrics, 35:351-362, 1993. URL https: //doi . org/ 10.2307/1270269. [p56]

R. D. Cook and S. Weisberg. Residuals and Influence in Regression. Chapman and Hall, 1982. [p56]

P. J. Diggle, P. J. Heagerty, K.-Y. Liang, and S. L. Zeger. Analysis of Longitudinal Data. Oxford University Press, 2002. [p68]

B. Efron. Better bootstrap confidence intervals. Journal of the American Statistical Association, 82:171-185, 1987. URL https://doi.org/10.2307/2289144. [p59]

M. Ezekiel. A method of handling curvilinear correlation for any number of variables. Journal of the American Statistical Association, 19:431-453, 1924. URL https://doi .org/10.2307/2281561. [p58]

J. Fox. Effect displays in R for generalised linear models. Journal of Statistical Software, 8:1-27, 2003. URL https://doi.org/10.18637/jss.v008.i15. [p57]

J. Fox and S. Weisberg. An R Companion to Applied Regression. Sage, Thousand Oaks CA, 2nd edition, 2011. [p57]

P. M. Grambsch and T. M. Therneau. Proportional hazards tests and diagnostics based on weighted residuals. Biometrika, 81:pp. 515-526, 1994. URL https://doi.org/10.1093/biomet/81.3.515. [p56]

F. Harrell. Regression Modeling Strategies: With Applications to Linear Models, Logistic and Ordinal Regression, and Survival Analysis. Springer-Verlag, 2015. [p57]

T. Hastie, R. Tibshirani, and J. Friedman. The Elements of Statistical Learning: Data Mining, Inference, and Prediction. Springer-Verlag, 2009. [p57]

T. J. Hastie and R. J. Tibshirani. Generalized Additive Models. Chapman \& Hall/CRC, 1990. [p56]

D. W. Hosmer and S. Lemeshow. Applied Logistic Regression. John Wiley \& Sons, 2000. [p66]

P. E. Johnson. Rockchalk: Regression Estimation and Presentation, 2016. R package version 1.8.101. [p57] 
M. Kutner, C. Nachtsheim, J. Neter, and W. Li. Applied Linear Statistical Models. McGraw-Hill, 2004. [p58]

J. M. Landwehr, D. Pregibon, and A. C. Shoemaker. Graphical methods for assessing logistic regression models. Journal of the American Statistical Association, 79:61-71, 1984. URL https://doi .org/10. 2307/2288334. [p67]

W. A. Larsen and S. J. McCleary. The use of partial residual plots in regression analysis. Technometrics, 14:781-790, 1972. URL https://doi.org/10.2307/1267305. [p58]

C. Loader. Locfit: Local Regression, Likelihood and Density Estimation., 2010. R package version 1.5-6. [p66]

A. Loy and H. Hofmann. Diagnostic tools for hierarchical linear models. Wiley Interdisciplinary Reviews: Computational Statistics, 5:48-61, 2013. URL https://doi .org/10.1002/wics.1238. [p56]

C. L. Mallows. Augmented partial residuals. Technometrics, 28:313-319, 1986. URL https: //doi .org/ 10.2307/1268980. [p56]

D. Meyer, E. Dimitriadou, K. Hornik, A. Weingessel, and F. Leisch. E1071: Misc Functions of the Department of Statistics, Probability Theory Group (Formerly: E1071), TU Wien, 2017. R package version 1.6-8. [p67]

R. J. O'Hara Hines and E. M. Carter. Improved added variable and partial residual plots for the detection of influential observations in generalized linear models. Journal of the Royal Statistical Society C, 42:pp. 3-20, 1993. URL https://doi .org/10.2307/2347405. [p67]

D. Pregibon. Logistic regression diagnostics. The Annals of Statistics, 9:705-724, 1981. URL https: //doi.org/10.1214/aos/1176345513. [p56,67]

G. Ridgeway. Gbm: Generalized Boosted Regression Models, 2017. R package version 2.1.3. [p68]

D. Sarkar. Lattice: Multivariate Data Visualization with R. Springer-Verlag, 2008. [p62]

T. Therneau. A Package for Survival Analysis in S, 2012. R package version 2.36-12. [p65]

W. N. Venables and B. D. Ripley. Modern Applied Statistics with S. Springer-Verlag, 2002. [p65]

H. Wickham. Ggplot2: Elegant Graphics for Data Analysis. Springer-Verlag, 2009. [p63]

C. S. Withers and S. Nadarajah. Improved confidence regions based on edgeworth expansions. Computational Statistics \& Data Analysis, 56:4366 - 4380, 2012. URL https://doi . org/10.1016/j. csda.2012.03.019. [p59]

F. S. Wood. The use of individual effects and residuals in fitting equations to data. Technometrics, 15: 677-695, 1973. URL https://doi.org/10.2307/1267381. [p58]

S. Wood. Mgcv: Mixed GAM Computation Vehicle with GCV/AIC/REML Smoothness Estimation, 2012. R package version 1.7-17. [p66]

S. N. Wood. Generalized Additive Models: An Introduction with R. Chapman \& Hall/CRC, 2006. [p56]

Patrick Breheny

Department of Biostatistics

University of Iowa

ORCiD: 0000-0002-0650-1119

patrick-breheny@uiowa.edu

Woodrow Burchett

Department of Statistics

University of Kentucky

woodrow. burchett@uky. edu 